\title{
La simulación como estrategia metodológica en la Facultad de Educación de la Universidad del País Vasco
}

\author{
Simulation as a Methodological Strategy in the Faculty of Education at the University of the \\ Basque Country
}

\section{Simulação como estratégia metodológica na faculdade de educação da Universidade do País Basco}

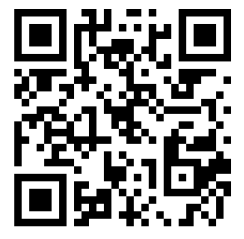

Zuriñe Gaintza-Jauregi

Universidad del País Vasco

Bilbao, España

zuri.gaintza@ehu.eus

(iD http://orcid.org/0000-0002-6192-0455

Recibido • Received • Recebido: 20 / 10 / 2018

Corregido • Revised • Revisado: 17 / 03 / 2020

Aceptado • Accepted • Aprovado: 21 / 07 / 2020

\begin{abstract}
Resumen: En este artículo subyace la idea de que se aprende haciendo. En concreto, esta investigación recoge el proceso de implementación y evaluación de una experiencia educativa innovadora en el ámbito universitario, basada en la simulación. El objetivo de la investigación es demostrar la eficacia de la simulación como estrategia metodológica en los estudios universitarios de magisterio en educación primaria. Para ello, al estudiantado universitario se le dota de la información teórica y práctica, sobre la asignatura Bases pedagógicas de la escuela inclusiva y sobre simulación y técnicas de enseñanza. Con ello, durante una sesión de la asignatura, estudiantes simulan una sesión tutorial para un curso de educación primaria en torno al tema de la inclusión. Para determinar los resultados en cuanto a adquisición de conocimiento se formula una pregunta en un ejercicio de evaluación, y para la valoración de la simulación cada discente completa un cuestionario. En los resultados se observa cómo la simulación lleva al estudiantado a asumir el papel de maestro o maestra de primaria; además, en su rol de profesor o profesora ha adquirido el contenido del tema, y desarrolla competencias y habilidades sociales, personales y profesionales. El estudio concluye, por lo tanto, que la simulación resulta eficaz como estrategia metodológica en los estudios universitarios de magisterio en educación primaria y que constituye en sí misma, una experiencia de innovación educativa.
\end{abstract}

Palabras claves: Universidad; metodología; estrategia; simulación; innovación. 
http://doi.org/10.15359/ree.24-3.11

http://www.una.ac.cr/educare

educare@una.ac.cr

\begin{abstract}
This article is based on the idea that we learn by doing. Specifically, this research includes the implementation and evaluation process, at the university, of an innovative educational experience based on simulation. The research aims to demonstrate the effectiveness of simulation as a teaching tool at the university teaching studies in primary education. To achieve this goal, university students receive theoretical and practical information on Pedagogical bases of the inclusiveschooland simulation and teaching techniques. With this, during a course session, the students simulate a tutorial session around the topic of inclusion for a primary school class. A question is asked in an evaluation exercise to determine the results in terms of knowledge acquisition, and, for the simulation assessment, the university students complete a questionnaire. The results show how simulation leads students to assume the role of a primary school teacher, and in their role as a teacher, they acquire the content of the topic and develop social, personal, and professional skills and abilities. The study, therefore, concludes that simulation is an effective methodological strategy in the university studies to train primary education teachers, and it constitutes, in itself, an educational innovation experience during these studies.
\end{abstract}

Keywords: University; methodology; strategy; simulation; innovation.

Resumo: O presente artigo se fundamenta na ideia do aprender fazendo. Especificamente, esta pesquisa inclui o processo de implementação e avaliação de uma experiência educacional inovadora no ambiente universitário, baseada na simulação. O objetivo da pesquisa é demonstrar a eficácia da simulação como estratégia metodológica nos cursos universitarios de ensino fundamental. Para isso, os estudantes universitários recebem informações teóricas e práticas, sobre o assunto Bases pedagógicas da escola inclusiva e sobre técnicas de simulação e ensino. Com isso, durante uma sessão do tema, os alunos simulam uma sessão tutorial para um curso de educação primária sobre o tema da inclusão. Para determinar os resultados em termos de aquisição de conhecimento, é realizada uma pergunta em um exercício de avaliação e, para a avaliação da simulação, cada estudante preenche um questionário. Os resultados mostram como a simulação leva os estudantes a assumir o papel de professor da escola primária e, em seu papel de professor, adquirir o conteúdo da disciplina e desenvolver compentencias e habilidades sociais, pessoais e profissionais. Portanto, o estudo conclui que a simulação é eficaz como estratégia metodológica nos estudos universitários de ensino fundamental e que constitui, por si só, uma experiência de inovação educacional nesses estudos.

Palavras-chaves: universidade; metodologia; estratégia; simulação; inovação.

\title{
Introducción
}

El Espacio Europeo de Educación Superior (EEES) supone, en los centros universitarios, un profundo cambio, no solo en cuanto a la elaboración de nuevos planes de estudio, sino también en cuanto a las trasformaciones de índole pedagógica que repercuten directamente en la docencia. El esquema tradicional de clase magistral se sustituye por el empleo de metodologías activas, que inciten al estudiantado a ser activo y curioso en su aprendizaje (Blumenfeld, et al., 1991). Sin duda, este cambio conlleva el desarrollo de nuevas competencias profesionales, orientadas ahora a conseguir que el alumnado participe en su proceso de enseñanza-aprendizaje. Así pues, el 
http://doi.org/10.15359/ree.24-3.11

profesorado docente e investigador (PDI) se encuentra ante una tarea tan novedosa como difícil: el conocimiento y uso de metodologías activas. Ante esta nueva realidad, el PDI debe recomponer su quehacer didáctico y convertir al estudiantado en el protagonista de su educación. Con ello, se hace inminente cambiar la mirada en el modo de instruir, ya no se trata de impartir conocimientos a través de una asignatura, sino de generar aprendizaje desde ella misma.

El PDI debe analizar los contenidos, los objetivos y las competencias que se quieren desarrollar para reflexionar sobre la instrucción más eficaz para cada tema en particular. En síntesis, el a priori de cada tema, para generar un aprendizaje mediado, ha de estar mucho más elaborado. Es en el antes, en el previo, en esa reestructuración de la materia, donde el PDI entra en contacto con diferentes metodologías. De ahí que, tal y como señala Alcoba (2012), la preocupación por la instrucción trae a un primer plano la investigación sobre las metodologías docentes. En el ámbito universitario, la investigación resalta el uso de diferentes estrategias dentro de las denominadas metodologías activas: aprendizaje basado en problemas, método del caso, contrato de aprendizaje y trabajo por pares, entre otras. La elección de usar una u otra está determinada por variables tales como: el contenido a trabajar; las condiciones de aprendizaje (ratio, tiempo de la sesión o espacio disponible); los objetivos y competencias ya marcados en el plan de estudio, y, sobre todo, el conocimiento o dominio que el personal docente tenga de la estrategia en cuestión. Es este personal docente quien, conocedor de que "cada método es bueno para determinadas situaciones de [enseñanza aprendizaje], pero ningún método es bueno para todas" (Fernández March, 2006, p. 41), opta por aquel que considera más apropiado.

En cuanto al estudiantado de magisterio se sabe que acepta bien las metodologías activas (Pazos Couto et al., 2015). Por ello, en el grado de magisterio en Educación Primaria, el tema 1 de la asignatura, Bases Pedagógicas de la Escuela Inclusiva, se imparte desde la premisa aprender haciendo utilizando la simulación como estrategia pedagógica. En este sentido, si bien, no existe ninguna experiencia similar en los estudios de magisterio, diferentes razones consolidan el uso de dicha estrategia:

Por un lado, los contenidos del tema 1 son de carácter teórico, y hay muchos datos que los grupos discentes pueden ser capaz de comprender a través del propio trabajo personal dirigido por su docente. Por otro lado, para la simulación de un aula de educación primaria, se requiere que el estudiantado de magisterio coja tanto el rol del escolar como el del maestro o maestra de escuela. Considerando las experiencias educativas y la práctica que estos grupos discentes universitarios han tenido, cabe esperar que el alumnado de magisterio no presente ninguna dificultad en desarrollar bien esos roles, pues las prácticas en escuelas ya han tenido lugar y conocen cómo se comportan y qué características poseen tanto los niños y las niñas de diferentes edades como el profesorado de los diferentes cursos. Además, la investigación señala que la simulación es "un procedimiento didáctico que implica, motiva y favorece aprendizajes significativos en el aula universitaria" (Navaridas Nalda, 2004, p. 63). Por todo ello, no se duda en llevar a cabo una experiencia de innovación educativa con dicha estrategia didáctica. 
http://doi.org/10.15359/ree.24-3.11

http://www.una.ac.cr/educare

educare@una.ac.cr

En lo que respecta al concepto de simulación, Gough y Whitehall (1962) lo definen como el proceso de diseñar un modelo de un sistema real y desarrollar experiencias con el objetivo de comprender el comportamiento de los elementos del sistema. De esta forma, en la medida en que es posible crear un ambiente simulado, es posible producir aprendizaje acerca de situaciones reales. Dos décadas más tarde, Brown et al. (1989) argumentan que el conocimiento se produce en situación y se define como una estrategia docente que reemplaza o amplía experiencias reales por experiencias guiadas. Estas experiencias simulan un contexto natural donde sus participantes se familiarizan con diferentes realidades; se facilitan los aprendizajes orientados tanto a la comprensión como a la utilización de nuevos mecanismos. En el ámbito universitario se ha visto que la simulación constituye una herramienta metodológica eficaz que potencia el interés, aumenta la motivación y facilita el desarrollo de competencias (Fernández March, 2006), de ahí que su uso esté muy expandido. Por ejemplo: en los estudios universitarios de derecho (Fach Gómez y Rengel, 2014); en los de medicina (Galindo López y Visbal Spirko, 2007) y enfermería (Urra Medina et al., 2017); y en los de ingeniería (Bodnar et al., 2016), entre otros.

Como se puede observar, el uso de la simulación como estrategia de instrucción no es nuevo, y la eficacia de su utilización en diferentes titulaciones queda patente en diversos estudios. Si bien existe un estudio que demuestra la eficacia de la simulación con estudiantado de magisterio en Educación Física (Rojas et al., 2015), no hay estudios con estudiantado de Magisterio en Educación Primaria, por lo que este trabajo constituye un referente para avanzar en la aplicación de nuevas metodologías en dicha especialidad. Con ello, esta investigación recoge el proceso de implementación y evaluación de una experiencia educativa innovadora en el ámbito universitario, basada en la simulación que, en este caso, se sustenta en el aprendizaje basado en proyectos. "el método de proyectos ... hace hincapié en la unificación del aprendizaje teórico y práctico, [en la] colaboración de alumnos y [en la inclusión de] elementos de la vida cotidiana en las instituciones de educación" (Huber, 2008, p. 73). Esto es, la técnica permite que un grupo de discentes articule diferentes actividades formativas y genere un proyecto de intervención profesional (Fernández March, 2006) que aumenta la motivación del alumnado, así como los resultados de aprendizaje (Ilter, 2014).

Ambos términos, simulación y proyectos, nos acercan a la globalidad de la estrategia de los proyectos en situación definida por Díaz Barriga Arceo (2005, p. 30), quien lo señala como "un aprendizaje eminentemente experiencial, pues se aprende al hacer y al reflexionar sobre lo que se hace en contextos de prácticas situadas y auténticas". En sí mismo, es un aprendizaje orientado a la acción, no se trata solo de aprender acerca de algo sino de hacer algo.

Con todo, el objetivo de la investigación es demostrar la eficacia de la simulación como estrategia metodológica en los estudios universitarios de magisterio en Educación Primaria, tanto para la adquisición de destrezas generalizables al contexto profesional como para la comprensión de contenidos. 
http://doi.org/10.15359/ree.24-3.11

\section{Método}

Se trata de un estudio descriptivo-interpretativo con intervención participante, ya que es la propia profesora quien diseña e implementa la intervención, recoge los datos, los analiza y los triangula. En relación con esta última tarea, se realiza una entrevista en torno a las conclusiones obtenidas en el estudio al estudiantado que durante la simulación desempeñó el rol de docente. Con todo, la función de este estudio es evaluativa y formativa, pues pretende obtener información relevante y útil de cara a mejorar la propia práctica docente. Con todo, esta investigación tiene un enfoque cualitativo y cuantitativo. Así, a través de preguntas abiertas se recoge el conocimiento adquirido del tema y, con un cuestionario, la opinión del estudiantado sobre la simulación como estrategia docente.

\section{Muestra}

De los 200 discentes con matrícula en el $2^{\circ}$ curso de grado en Educación Primaria en el campus de Bizkaia de la Universidad del País Vasco en la asignatura Bases Pedagógicas de la Escuela Inclusiva, participan 141 (95 mujeres y 46 hombres) de los dos grupos cuya lengua de aprendizaje es el vasco (denominados 31 y 32 ).

Para la selección de este grupo de participantes se utilizó la técnica de muestreo no probabilístico por conveniencia, ya que se escogió, de manera intencionada, estudiantado que cursaba la asignatura de Bases Pedagógicas de la Escuela Inclusiva. Se informa a los sujetos participantes de la existencia de este estudio y se les solicita su permiso para las posibles grabaciones de las sesiones.

\section{Procedimiento}

Dado que este estudiantado universitario se está formando para ser docentes en los niveles educativos de educación primaria (6-12 años), para la elaboración y desarrollo del proyecto, se le orienta en las posibles técnicas que pueden usar para realizar una simulación en dichos niveles. En este sentido, cada simulación va a ser única y específica, tanto por el curso de educación primaria al que se dirija como por la técnica que cada grupo elija. Con la simulación, el alumnado de Magisterio se sitúa como futuro docente de educación primaria. Esta estrategia implica dos momentos bien diferenciados en el tiempo: uno, la reflexión grupal previa para la elección de la técnica más apta en función de la edad de sus escolares; y dos, la simulación, la puesta en práctica en el aula universitaria.

\section{Implementación}

La asignatura, en los grupos 31 y 32, se divide por semanas en una sesión teórica de 3 horas y en otra práctica de 1 hora y 30 minutos, en la que los grupos se dividen en 4 grupos prácticos: 31A, 31B, 32A y 32B. En cada grupo práctico, se forman pequeños grupos (entre 4-8 discentes). Un total de 
http://doi.org/10.15359/ree.24-3.11

http://www.una.ac.cr/educare

educare@una.ac.cr

22 mini-grupos (mn-grs) constituyen las unidades sistémicas para la simulación (véase Tabla 2). Para la elaboración de la simulación, se utilizan tanto las sesiones teóricas, como las prácticas, además, es necesario que el estudiantado realice un trabajo complementario en sus horas personales.

En la primera sesión teórica se presenta el tema Historia y evolución de la educación especial y se indican cuáles son los documentos de lectura obligatoria (Monzón, 2011; Núñez Mayán, 2008; Orcasitas, 2005). Tras una breve exposición, se explica el procedimiento para la simulación. En este sentido, se les dice que, cada mn-gr tiene que preparar para un curso de primaria una sesión sobre alguno de estos temas: la evolución de la educación especial a lo largo de la historia o, el concepto de escuela inclusiva. Para que visualicen bien la idea, se insiste en que el día de la simulación, cada mn-gr actuará en función del curso elegido, y el resto del alumnado se comportará como escolares de ese curso, con lo cual se transformará el aula universitaria en un aula de primaria.

Como ya se ha dicho, este estudiantado ha realizado las prácticas en las escuelas, por ello, se les propone que recuerden ese periodo y compartan con sus compañeros y compañeras tanto las características de sus escolares como el tipo de técnicas observadas. Después, cada mn-gr acuerda un ciclo y un curso. En la medida de lo posible se respeta la elección de cada mngr siempre y cuando estén presentes todos los ciclos de primaria. Con esto, tras la simulación se ven las diferencias y similitudes entre impartir un contenido en el $1^{\circ}$ ciclo (6-8 años) o hacerlo en el $3^{\circ}$ ciclo (10-12 años). La sesión finaliza con el cronograma de la simulación (Tabla 1).

Tabla 1: Cronograma para la simulación

\begin{tabular}{|c|c|c|c|c|c|c|c|c|c|c|}
\hline \multirow[b]{2}{*}{ FASES } & \multirow[b]{2}{*}{ ACTIVIDADES } & \multicolumn{9}{|c|}{ SEMANAS (Teoría / Práctica) } \\
\hline & & $1^{\circ} \mathrm{T}$ & $1 \mathrm{oP}$ & $2^{\circ} \mathrm{T}$ & $2 \circ \mathrm{P}$ & $3^{\mathrm{a} T}$ & $3 \mathrm{ap}$ & $4^{\mathrm{a} T}$ & $4 \mathrm{OP}$ & $5^{\circ}$ \\
\hline \multirow{2}{*}{ Teoría } & Explicación & & & & & & & & & \\
\hline & Documentos obligatorios & & & & & & & & & \\
\hline \multirow{2}{*}{ Elección } & Ciclo/curso & & & & & & & & & \\
\hline & Técnica & & & & & & & & & \\
\hline \multirow[t]{2}{*}{ Revisión teórica } & $\begin{array}{l}\text { Características de escolares y } \\
\text { metodologías }\end{array}$ & & & & & & & & & \\
\hline & Técnica elegida & & & & & & & & & \\
\hline \multirow{2}{*}{ Elaboración } & Trabajo escrito & & & & & & & & & \\
\hline & Montaje simulación & & & & & & & & & \\
\hline Simulación & Presentación & & & & & & & & & \\
\hline Valoración & Rellenar cuestionario & & & & & & & & & \\
\hline
\end{tabular}

Nota: Elaboración propia. 
http://doi.org/10.15359/ree.24-3.11

En la segunda sesión cada mn-gr elige la técnica. Las siguientes sesiones se dedican a la lectura, resumen y adaptación de los documentos obligatorios, así como al diseño de la simulación. Tras un mes de trabajo individual y grupal en el que la profesora de la asignatura tiene una intervención mediadora, orientada tanto a potenciar el aprendizaje autónomo como la participación activa, se realiza la simulación.

En cuanto a la evaluación, la profesora de la asignatura la realiza teniendo en cuenta cuatro factores: (1) las anotaciones tomadas a lo largo de las 4 semanas; (2) el trabajo escrito elaborado con los documentos obligatorios; (3) las anotaciones recogidas durante la simulación que consideran la adaptación a la edad del grupo de escolares: el vocabulario, la velocidad, la expresión, el contenido y el material utilizado; y (4) la participación del estudiantado durante las simulaciones. Tal y como otros estudios resaltan, la evaluación individual de cada estudiante no resulta nada fácil, pues no todas las personas se centran en las mismas tareas y éstas no tienen el mismo grado de dificultad (Tejada Fernández, 2010).

\section{Instrumentos}

Por un lado, para recoger los datos referidos al análisis y valoración de la implementación de la simulación, en la siguiente clase a la simulación, se pasa a todo el grupo un cuestionario de evaluación creado ad hoc. Este cuestionario es sometido a un proceso de validación a través de la consulta a un panel de jueces compuesto por cinco personas expertas del área, que cumplen con el criterio de al menos cinco años de experiencia (Perroca, 2011). Tras recoger sus aportaciones, finalmente, el propio panel experto da por bueno el cuestionario que cuenta con las características que a continuación se describen. Presenta dos partes bien diferenciadas: una cuantitativa y otra cualitativa.

En la primera, con el fin de conocer la opinión del estudiantado sobre la simulación como estrategia docente, los sujetos participantes muestran su grado de acuerdo o desacuerdo sobre cinco ítems en una escala tipo Likert del 1 al 5. A mayor puntuación valoración más positiva sobre la simulación. Ítems:

- ¿Has comprendido los contenidos teóricos?

- ¿Han sido suficientes las orientaciones docentes?

- ¿La repetirías?

- ¿Consideras esta estrategia útil?

- ¿Cuál es tu valoración general de la técnica? 
http://doi.org/10.15359/ree.24-3.11

http://www.una.ac.cr/educare

educare@una.ac.cr

En la segunda, con el fin de conocer la percepción del estudiantado bien de la simulación, bien de la adquisición de destrezas no conceptuales, se recogen datos de tipo cualitativo a través de tres preguntas abiertas:

- Para ti, ¿qué ha sido lo más difícil?

- Para ti, ¿qué ha sido lo que más te ha gustado?

- Además de los contenidos teóricos del tema, ¿crees que has aprendido algo más con esta técnica? ¿Qué?

Por otro lado, para determinar que la simulación es válida para la adquisición de los contenidos de carácter teórico del tema 1, en el ejercicio de evaluación se formula una pregunta abierta: Describe brevemente cuál ha sido la evolución de la educación especial a lo largo de la historia. La pregunta se corrige y se evalúa por la docente de la asignatura de acuerdo con los contenidos del tema y su puntuación se recoge en el análisis de la información de este estudio.

\section{Análisis y resultados}

Los datos que se recogen tras la simulación se analizan aplicando una metodología mixta que integra una parte cuantitativa y otra cualitativa. En cuanto a la cuantitativa, las respuestas obtenidas en la escala se analizan utilizando el SPSS, Versión 18.0.0. Tras comprobar que la muestra sigue una distribucional normal para la variable valoración (KS: 0.452; sig.: .512) se realizan análisis descriptivos de la tendencia central (medias), así mismo, se realizan pruebas análisis de varianza (ANOVA) y pruebas Test-t con las puntuaciones de los ítems con respecto a las variables de género, ciclo y técnica. El nivel límite para determinar diferencias significativas se establece en $\mathrm{p}<0.05$. En la Tabla 2 se observa el número de participantes, así como el curso y la técnica elegida por cada mn-gr. La valoración media sobre la simulación de cada mn-gr se recoge en la última columna.

Como se recoge en la Tabla 2, el alumnado selecciona 7 técnicas. En concreto, $6 \mathrm{mn}$ grs realizan un video, dos dirigidos al $3^{\circ}$ ciclo de primaria y cuatro al $2^{\circ} ; 5$ mn-grs elaboran un cuento, cuatro dirigidos al $1^{\circ}$ ciclo y uno al $2^{\circ} ; 3$ mn-grs montan un teatro, uno en cada ciclo; otros $3 \mathrm{mn}$-grs construyen un puzle, uno dirigido al $2^{\circ}$ ciclo y dos al $3^{\circ} ; 2 \mathrm{mn}$-grs proponen un juego, tres dirigidos al $3^{\circ}$ ciclo y dos al $2^{\circ}$; también 2 mn-grs realizan un PowerPoint, uno para el $1^{\circ}$ ciclo y otro para el $3^{\circ}, y$, finalmente, un mn-gr opta por el diseño de un mural para el $2^{\circ}$ ciclo. La valoración de la técnica sobre 5 es: media $=4,12$. Es decir, realizan una valoración muy positiva de la técnica. 
http://doi.org/10.15359/ree.24-3.11

http://www.una.ac.cr/educare educare@una.ac.cr

Tabla 2: Técnica y curso elegido por cada mn-gr y valoración sobre la simulación

\begin{tabular}{|c|c|c|c|c|c|c|c|c|c|}
\hline Grupo & & Mn-gr & $\begin{array}{c}\text { Participantes } \\
\text { (hombres+mujeres) }\end{array}$ & Curso & Técnica & $\begin{array}{l}\text { Media } \\
\text { escalar }\end{array}$ & $\begin{array}{c}\text { Desviación } \\
\text { típica }\end{array}$ & Min. & Max \\
\hline \multirow[t]{12}{*}{31} & AA & G1 & $0+6$ & $4^{\circ}$ & Puzle de dibujos & 4,33 & ,516 & 4 & 5 \\
\hline & & G2 & $4+1$ & $5^{\circ}$ & Juego inclutrivial & 4,20 & ,447 & 4 & 5 \\
\hline & & G3 & $1+4$ & $3^{\circ}$ & Video & 4,00 & 707 & 3 & 5 \\
\hline & $A$ & G4 & $6+0$ & $1^{\circ}$ & Cuento & 4,33 & ,516 & 4 & 5 \\
\hline & & G5 & $3+4$ & $1^{\circ}$ & Cuento & 4,00 & ,000 & 4 & 4 \\
\hline & & G6 & $0+6$ & $6^{\circ}$ & P.Point+ejercicio & 4,42 & ,492 & 4 & 5 \\
\hline & B & G7 & $1+5$ & $3^{\circ}$ & Teatro & 4,50 & ,548 & 4 & 5 \\
\hline & & G8 & $0+5$ & $4^{\circ}$ & Video con cuento & 4,00 & ,000 & 4 & 4 \\
\hline & B & G9 & $0+5$ & $1^{\circ}$ & Cuento & 3,80 & ,447 & 3 & 4 \\
\hline & & G10 & $9+0$ & $6^{\circ}$ & Teatro & 4,33 & ,500 & 4 & 5 \\
\hline & & G11 & $0+5$ & $2^{\circ}$ & P. Point cuento & 4,60 & ,548 & 4 & 5 \\
\hline & & G12 & $0+5$ & $6^{\circ}$ & Puzle de texto & 3,80 & ,447 & 3 & 4 \\
\hline \multirow[t]{10}{*}{32} & $A$ & G1 & $1+7$ & $4^{\circ}$ & Cuento & 4,00 & ,000 & 4 & 4 \\
\hline & & G2 & $6+1$ & $1^{\circ}$ & Cuento & 4,00 & ,000 & 4 & 4 \\
\hline & & G3 & $2+6$ & $6^{\circ}$ & Puzle de texto & 3,87 & ,354 & 3 & 4 \\
\hline & & G4 & $1+6$ & $2^{\circ}$ & Juego animales & 4,00 & ,000 & 4 & 4 \\
\hline & & G5 & $6+0$ & $4^{\circ}$ & Video & 4,17 & ,408 & 4 & 5 \\
\hline & B & G6 & $2+4$ & $6^{\circ}$ & Video (entrevista) & 4,33 & ,516 & 4 & 5 \\
\hline & & G7 & $2+4$ & $6^{\circ}$ & Video & 4,17 & ,408 & 4 & 5 \\
\hline & & G8 & $0+9$ & $4^{\circ}$ & Mural & 4,11 & 333 & 4 & 5 \\
\hline & & G9 & $2+5$ & $4^{\circ}$ & Video & 4,00 & ,000 & 4 & 4 \\
\hline & & G10 & $0+7$ & $1^{\circ}$ & Teatro & 3,86 & 378 & 3 & 4 \\
\hline
\end{tabular}

Nota: Elaboración propia.

Destaca que la mayoría de los mn-gr que realiza la simulación para el $1^{\circ}$ ciclo se decanta por la realización de un cuento (85,7\%). Si bien el formato de éste cambia (DIN A- 4 plastificado o no; folios, cartulina, PowerPoint), en todos aparecen animales (vacas moradas, elefantes sin trompa, cocodrilos sin dientes, etc.). Incluso el teatro para ese ciclo narra la historia de un pájaro que no puede volar. También se ve que $6 \mathrm{mn}$-grs (que suman el 24,8 \% de participantes) crean videos de variado contenido y forma: historias, sketches, cuento y entrevistas.

En la Tabla 3, se recoge el número de participantes en las diferentes técnicas por ciclos $-1{ }^{\circ}$ ciclo (6-8 años); $2^{\circ}$ ciclo (8-10 años) y $3^{\circ}$ ciclo (10-12 años)- , los sujetos participantes en cada técnica y se atiende el género y la valoración en función de la técnica utilizada. 
http://doi.org/10.15359/ree.24-3.11

http://www.una.ac.cr/educare

educare@una.ac.cr

Tabla 3: Distribución de las técnicas en función del ciclo y media de valoración de la simulación según técnica

\begin{tabular}{lccccc}
\hline & & Ciclo & & Participantes & Media valoración \\
\hline Técnica & 10 & $2^{\circ}$ & $3^{\circ}$ & (hombres+mujeres) & escala Likert \\
\hline Puzle & 0 & 6 & 13 & $2+17$ & 4,00 \\
Juego & 7 & 0 & 5 & $5+7$ & 4,08 \\
Video & 0 & 23 & 12 & $13+22$ & 4,11 \\
Cuento & 25 & 8 & 0 & $16+17$ & 4,03 \\
Teatro & 7 & 6 & 9 & $10+12$ & 4,23 \\
Mural & 0 & 9 & 0 & $0+9$ & 4,11 \\
PowerPoint & 5 & 0 & 6 & $0+11$ & 4,55 \\
\hline
\end{tabular}

Nota: Elaboración propia.

En los análisis de comparación de medias (Test-t y ANOVA) no se observan diferencias significativas en relación con el género o el ciclo. La valoración es relativamente alta tanto para las alumnas como para los alumnos, y lo mismo ocurre en cuanto al ciclo elegido para la simulación. Sin embargo, sí aparecen diferencias significativas en cuanto a la técnica que eligen y la valoración que hacen: quienes aplican el PowerPoint valoran más positivamente la técnica (Tabla 4).

Tabla 4: Diferencias en la valoración de la simulación según técnica elegida

\begin{tabular}{|c|c|c|c|c|c|c|c|c|c|c|c|}
\hline \multirow[t]{2}{*}{ Técnica } & \multirow[t]{2}{*}{$\mathrm{N}$} & \multirow[t]{2}{*}{ Media } & \multirow[t]{2}{*}{ Desv. est. } & \multirow[t]{2}{*}{ Error est. } & \multicolumn{3}{|c|}{$95 \%$ del interv. de conf. para la media } & \multirow[t]{2}{*}{ Mín. } & \multirow[t]{2}{*}{ Máx. } & \multicolumn{2}{|c|}{ Anova } \\
\hline & & & & & Límite inf. & & Límite sup. & & & $\mathrm{F}$ & Sig. \\
\hline Puzle & 19 & 4,00 & ,471 & 108 & 3,77 & 4,23 & & 3 & 5 & & \\
\hline Juego & 12 & 4,08 & ,289 & ,083 & 3,90 & 4,27 & & 4 & 5 & & \\
\hline Vídeo & 35 & 4,11 & ,404 &, 068 & 3,98 & 4,25 & & 3 & 5 & & \\
\hline Cuento & 33 & 4,03 & ,305 &, 053 & 3,92 & 4,14 & & 3 & 5 & 2,723 & ,016 \\
\hline Teatro & 22 & 4,23 &, 528 & 113 & 3,99 & 4,46 & & 3 & 5 & & \\
\hline Mural & 9 & 4,11 & 333 & 111, & 3,85 & 4,37 & & 4 & 5 & & \\
\hline PowerPoint & 11 & 4,55 &, 522 & 157 & 4,19 & 4,90 & & 4 & 5 & & \\
\hline
\end{tabular}

Nota: Elaboración propia.

\begin{tabular}{l|l}
\hline 10 & Zuriñe Gaintza-Jauregi
\end{tabular} 
http://doi.org/10.15359/ree.24-3.11

En cuanto a los datos cualitativos, tras preparar, transcribir y organizar las respuestas a las tres preguntas abiertas, se usa NVivo9 (QSR International, 2011) para codificar las unidades de información, y crear y estructurar la información en categorías. Las categorías en relación a cada pregunta fueron las siguientes.

\section{1) Para ti, ¿qué ha sido lo más difícil?}

Codificadas las respuestas de este ítem, surgen las siguientes categorías: adaptación del contenido, la simulación, el trabajo grupal, el trabajo individual, la técnica y otros. Tras el análisis se aprecia que para más del 50 \% la mayor dificultad es la adaptación de los documentos a escolares. Esta percepción la tienen 75 participantes y se refleja en frases como: gran desconocimiento de las capacidades del alumnado de primaria, para poder trasmitir bien los contenidos o hacerlos atractivos o tema demasiado profundo para el alumnado de primaria. La dificultad en la adaptación varía con el ciclo y aumenta para el $1^{\circ}$ ciclo; la entonación, la forma de atraer la atención de escolares, la velocidad de habla o lectura, la manera de contar, etc., son respuestas reiteradas a este ítem para este ciclo.

Un número más reducido, 21 participantes, encuentra la mayor dificultad en el trabajo grupal, acordar cómo encauzar la tarea, cómo organizarse y coordinarse o cómo repartir y distribuir los textos y crear historias constituyen momentos de conflicto. Por el contrario, para 22 , lo dificultoso es la lectura y el resumen de los documentos, esto es, el trabajo individual. En ocasiones, la dificultad está relacionada con la técnica: de 7 participantes que señalan ésta, 5 realizan un montaje de video. Por último, 9 discentes consideran que lo más difícil es la simulación al implementar un cuento o un teatro.

\section{2) Para ti, ¿qué ha sido lo que más te ha gustado?}

Una vez codificadas las respuestas a este ítem, surgen las siguientes categorías: la simulación, el diseño, el conocimiento de otras metodologías, el tema y otros. Tras el análisis destacan la simulación y la elaboración del proyecto. Así, un total de 50 discentes consideran la escenificación tanto del rol de docente como de escolares de primaria lo más atractivo y 43 participantes consideran que lo que más atractivo es el proceso de llevar la teoría a imágenes. Curiosamente, 14 participantes señalan ambas categorías, categorías recogidas en expresiones como: adecuar el contenido teórico al vídeo y presentarlo en el aula o preparar el cuento (dibujar, hacer la historia, contarlo...). Para 16 discentes, lo más interesante es conocer diferentes formas de impartir un mismo contenido, lo que lleva a analizar diferentes técnicas de instrucción aptas para el aula de primaria. Frases como lo que generalmente se imparte de manera tradicional ver qué existen otras formas de poder hacerlo más atractivas para los niños o ver modos tan distintos de trabajar un mismo tema, nos hacen pensar en un cambio de estructura de pensamiento a la 
http://doi.org/10.15359/ree.24-3.11

http://www.una.ac.cr/educare

educare@una.ac.cr

hora de instruir en un futuro. Para 8 discentes el contenido del tema la historia de la educación especial es, en sí mismo, motivante. Por último, también nos encontramos una respuesta que a la cual le parece divertido y con dos de quienes les gusta todo.

\section{3) Además de los contenidos teóricos del tema, ¿crees que has aprendido algo más con la simulación? ¿Qué?}

Después de codificar las respuestas a este ítem, surgen las siguientes categorías: nuevas técnicas de instrucción, adaptar contenido, rol docente, trabajar en grupo, técnica utilizada y otras. Tras el análisis se constata que 135 participantes consideran que han adquirido otra serie de conocimientos. En este sentido, 71 señalan que han aprendido nuevas y diferentes técnicas para instruir al alumnado de primaria. Otro grupo de 61 apuntan haber desarrollado habilidades para adaptar la información a los diferentes ciclos y 23 resaltan el comportamiento en su futuro rol docente. Como era de esperar, 50 ponen énfasis en el aprendizaje para el trabajo grupal, idea plasmada en expresiones como: trabajar en colaboración con los demás y aprender de ellos o valorar el trabajo de los demás. Un número también considerado de participantes, 33 en concreto, se centran en lo aprendido en cuanto a la técnica usada para la simulación. Cada técnica requiere de unos conocimientos y habilidades para su correcta adecuación, este aprendizaje se refleja cuando dicen: he aprendido cómo realizar una historia en teatro, qué personajes deben aparecer en un cuento y cómo dibujarlos o he aprendido a usar Movie Maker. Además, en menor cuantía, aparecen otros aprendizajes transversales como: quitar vergüenza, meterse en el papel del niño, reflexionar, improvisar, imaginar, etc.

De las anotaciones recogidas por la profesora del curso durante las simulaciones se destaca que todos los cuentos presentados para el $1^{\circ}$ ciclo tienen un vocabulario relativamente simple, claro y adecuado a los grupos escolares de esos cursos. Las historias son entretenidas, bonitas y fáciles $y$, sobre todo, giran en torno al tema planteado. También se observa esto en la simulación para el $2^{\circ}$ ciclo. Por otra parte, tanto el juego de inclutrivial como los puzles parten de un conocimiento previo del tema y, teniendo en cuenta que están dirigidos a escolares de 10-12 años, contienen demasiada información. Igualmente hay que poner en valor que algunos mn-gr han permitido dar voz a diferentes agentes de la comunidad educativa editando videos donde se entrevista a dos personas con ceguera: un adulto de 54 años y un escolar de 8 años.

Las anotaciones del PDI respecto a la participación del estudiantado en la simulación también son positivas. Cada mn-gr dirige la sesión y los compañeros y compañeras se comportan como infantes. Se destaca el comportamiento y las ganas con las que participan tanto en el juego de animales simulado como en el puzle, con este, el alumnado no cesa hasta finalizarlo implicándose en la tarea y realizando preguntas. 
http://doi.org/10.15359/ree.24-3.11

Por último, en cuanto a los resultados obtenidos del análisis de las puntuaciones en la pregunta de contenido del ejercicio de evaluación final hay que señalar que un total de 135 participantes supera la calificación de 5/10 puntos, donde la media del conjunto es de 8.12/10. Es decir, tras la simulación solo el 4,3 \% del alumnado no alcanza los conocimientos necesarios para dar por superado el tema.

\section{Discusión}

A través de esta experiencia educativa se ha querido indagar si la simulación es eficaz como recurso didáctico con estudiantado universitario de grado de Magisterio de Educación Primaria. Por un lado, se evalúa la adquisición de los contenidos del tema y se analiza el posible desarrollo de competencias y habilidades profesionales; y por otro, se analizan las técnicas elegidas para la simulación. Además, con base en la información obtenida, se obtienen conclusiones en cuatro campos: uno guarda relación con el contenido, otro con la estrategia didáctica, un tercero con la técnica que eligen y un cuarto con la labor del PDI.

1. En cuanto al contenido del tema, y en vista de la calificación en el ejercicio de evaluación final, se puede concluir que, en general, el estudiantado lo asimila, por lo que el uso de la simulación es eficaz para su adquisición.

2. Con respecto a la estrategia didáctica, se perciben cuatro aspectos concluyentes:

- Que se aprende haciendo y, sobre todo, actuando en situaciones cercanas a la realidad. La simulación sirve para que el alumnado se percate de la importancia que en la labor del profesorado tienen tanto la planificación, la metodología, como la propia instrucción.

- Que cada simulación es, en sí misma, un aprendizaje para su futuro profesional. Los sujetos participantes tienen la oportunidad de presenciar cómo se adapta un marco teórico con diferentes técnicas a distintos cursos. El futuro profesorado aprende que, en función de la situación de enseñanza-aprendizaje, es más conveniente el uso de una u otra técnica (Fernández March, 2006).

- Que con la simulación se mejoran las destrezas y relaciones interpersonales. La interacción que se genera en el trabajo grupal fomenta el desarrollo de habilidades sociales y personales (Robinson, 1993).

- Que para la simulación, entran en juego la imaginación y la creatividad. El alumnado diseña una técnica y evidencia su grado de capacidad creativa, imaginación y plasticidad mental. Tal y como concluye el Grupo Si(e)te.Educación (2012, p. 27) "la acción educativa no puede darse por comprendida sin prestar la consideración adecuada a la relación creatividad-educación-innovación". 
http://doi.org/10.15359/ree.24-3.11

http://www.una.ac.cr/educare

educare@una.ac.cr

3. La elección de la técnica nos lleva a tres conclusiones:

- Que para el estudiantado de Magisterio, el cuento resulta un camino fácil para motivar, entretener y, a la vez, trabajar conceptos en el $1^{\circ}$ ciclo de primaria (6-8 años). Aunque futuros estudios deberán confirmar estas hipótesis, a priori, esto puede deberse a tres factores: (a) que también en esas edades les contaron cuentos; (b) que lo han visto durante el período práctico y; (c) que conocen estudios que abogan por el cuento como recurso didáctico. La ventaja del cuento en primaria se encuentra en que la carga, explícitamente teórica, se presenta diluida en elementos de ocio y lúdicos (Jiménez Frías et al., 2001).

- Que con la simulación mucho estudiantado aumenta su competencia digital, competencia imprescindible en cualquier docente de primaria (Quintana Albalat, 2000). Aunque encuentran dificultades a la hora de preparar el contexto, resaltan haber aprendido a trabajar con editores de audio y vídeo como Movie Maker.

- Que los sujetos participantes que utilizan el PowerPoint son los que mejor valoran la simulación. Revisando la bibliografía científica observamos que hay razones que podrían explicar esta tendencia. Así, por un lado, se sabe que las presentaciones guiadas por ordenador son un recurso ampliamente extendido en niveles preuniversitarios (Johnson, 2008), hecho que a su vez lleva a que sea muy aceptado por el estudiantado (Tárraga Mínguez et al., 2012). Por otro lado, su proceso de elaboración, queda en manos de la persona que lo diseña (van Dijk, 1996). Quizá por todo ello, el alumnado de Magisterio está muy familiarizado con esta herramienta $y$, debido al margen de libertad que otorga, se siente seguro en la elaboración y confiado al utilizarla en una situación de simulación.

Con todo, cabe concluir que la simulación es una estrategia eficaz tanto para la comprensión y asimilación de contenidos, como para la adquisición de destrezas generalizables al contexto profesional para el estudiantado universitario de Magisterio de Educación Primaria. Con ello, tal y como recogen Martínez-Bello y Bernabé Villodre (2019), con este estudio se puede concluir, también, que la innovación educativa en la formación del profesorado no tiene por qué incluir el uso de las tecnologías de información y comunicación. Todos estos datos exigen ser sometidos a seguimiento en futuras investigaciones.

\section{En relación con la labor del personal docente universitario, cabe concluir:}

- Que el desarrollo de metodologías activas y, en particular la puesta en práctica de la simulación, supone al PDI un reto continuo. Tal y como concluye Huber (2008), el personal docente no solo tiene que estar formado y conocer su área, sino que, además, debe tener "aptitudes pedagógicas", es decir, conocimientos y destrezas 
http://doi.org/10.15359/ree.24-3.11

en el campo de la pedagogía, de la didáctica y de la psicología. Implementar metodologías activas como la aquí descrita supone estar permanentemente actualizado en estas áreas.

- Que el uso de este tipo de metodologías tiene un matiz de compromiso. La labor pedagógica y didáctica del personal docente transita más allá de la preparación de las clases teóricas, teniendo, la mayoría de las veces, que centrarse en las posibilidades de cada contenido para desarrollar en el estudiantado esas competencias y habilidades profesionales que superan el marco de su propia asignatura.

En síntesis, este estudio es innovador en cuanto al uso de la simulación con alumnado de Magisterio y demuestra que dicha estrategia es un procedimiento útil como recurso didáctico y herramienta de instrucción en la formación de futuros maestros y maestras. La simulación, en sí, se presenta como un puente que enlaza la teoría abstracta del aula con la realidad profesional concreta, por ello representa un mecanismo efectivo, práctico y eficaz para el futuro desempeño profesional del estudiantado. La simulación se plantea aquí como escenario para la adquisición de competencias y habilidades sociales, personales y profesionales. Con todo, se puede afirmar que esta estrategia se adapta al sistema propugnado por el EEES, en el cual se fomenta en el alumnado el desarrollo de competencias y habilidades necesarias como futuro personal docente de educación primaria.

Entre las principales limitaciones inherentes a esta investigación es preciso mencionar que, si bien la metodología ha permitido determinar hasta qué punto la simulación resulta eficaz como herramienta pedagógica en el grado de magisterio de educación primaria, los resultados se circunscriben al estudio de una realidad concreta y sujeta a un contexto determinado, lo que limita la transferibilidad de los resultados a otros contextos. Así mismo, a pesar de que el estudio ha contado con datos que van más allá de la mera opinión (como es la calificación obtenida por el alumnado en el tema objeto de aprendizaje: Historia y evolución de la educación especial), una parte importante de los datos se refieren a la valoración que realiza el alumnado sobre la utilidad de la simulación como herramienta de aprendizaje. Este hecho sitúa esta investigación en la esfera de estudios interpretativos y fenomenológicos en los cuales la percepción subjetiva es un elemento determinante a tener en cuenta en el alcance de este estudio.

Además, la simulación como estrategia educativa, en sí misma, no está exenta de limitaciones. Por un lado, aunque la simulación permite aproximarse a la realidad, nunca la representa de manera íntegra ni la reproduce de manera completa y, a juicio de muchos autores y autoras, este es su mayor inconveniente (Gómez, 2004). Por otro lado, en el caso de la simulación con discentes de magisterio, no solo se requiere compromiso docente, sino que resulta evidente que, además, el profesorado universitario conozca la realidad de aula de primaria, tanto las características y el comportamiento de sus escolares como diferentes técnicas de enseñanza. 
http://doi.org/10.15359/ree.24-3.11

http://www.una.ac.cr/educare

educare@una.ac.cr

\section{Transferencia a la práctica}

El estudio avala la idea de que, al igual que ocurre en otras titulaciones universitarias, en el grado de Magisterio de Educación Primaria, la simulación es también una estrategia eficaz. En este sentido, su propia práctica en el aula universitaria evidencia la posibilidad de transformarla en un aula de educación primaria en cuanto al uso metodológico y a la adquisición de los roles tanto de docentes como del alumnado. Además, el uso de la simulación trae a un primer plano el desarrollo de competencias profesionales a los futuros grupos docentes de primaria. Por ello, en al ámbito universitario, resulta necesario reflexionar sobre estrategias en el campo de las metodologías activas. En investigaciones futuras se podría implementar la simulación tanto en el grado en educación infantil como en el máster en educación secundaria.

\section{Referencias}

Alcoba González, J. (2012). La clasificación de los métodos de enseñanza en la educación superior. Contextos Educativos, 15, 93-106. https://doi.org/10.18172/con.657

Blumenfeld, P. C., Soloway, E., Marx, R. W., Krajcik, J. S., Guzdial, M. y Palincsar, A. (1991). Motivating project-based learning: Sustaining the doing, supporting the learning. Educational psychologist, 26(3-4), 369-398. https://doi.org/10.1080/00461520.1991.9653139

Bodnar, C. A., Anastasio, D., Enszer, J. A. y Burkey, D. D. (2016). Engineers at play: Games as teaching tools for undergraduate engineering students. Journal of Engineering Education, 105(1), 147-200. https://doi.org/10.1002/jee.20106

Brown, J. S., Collins, A. y Duguid, P. (1989). Situated cognition and the culture of learning. Educational researcher, 18(1), 32-42. https://doi.org/10.3102/0013189X018001032

Díaz Barriga Arceo, F. (2005). Enseñanza situada: Vínculo entre la escuela y la vida. McGraw Hill.

Fach Gómez, K. y Rengel, A. (2014). El aprendizaje a través de la simulación en el moot practice: Una estrategia docente para la mejora de la formación jurídica universitaria en el marco del EEES. REJIE. Revista Jurídica de Investigación e Innovación Educativa, 9, 23-48. http:// www.eumed.net/rev/rejie/09/index.htm

Fernández March, A. (2006). Metodologías activas para la formación de competencias. Educación Siglo XXI, 24, 35-56. https://revistas.um.es/educatio/article/view/152/135

Galindo López, J. y Visbal Spirko, L. (2007). Simulación, herramienta para la educación médica. Salud Uninorte, 23(1), 79-95. http://www.scielo.org.co/pdf/sun/v23n1/v23n1a09.pdf

Gómez, L. M. (2004). Entrenamiento basado en la simulación, una herramienta de enseñanza y aprendizaje. Revista colombiana de anestesiología, 32(3), 201-208. Recuperado de https:// www.redalyc.org/articulo.oa?id=195117787007 
http://doi.org/10.15359/ree.24-3.11

Gough V. E. yWhitehall S. G. (1962). Universal tyre test machine. En G. Eley (Ed.). Proceedings of the 9th International Automobile Technical Congress, discussion Fédération Internationale des Sociétés d'Ingénieurs des Techniques de IÁutomobile (FISITA) (pp. 117-137). Institution of Mechanical Engineers.

Grupo Si(e)te.Educación. (2012). Creatividad, educación e innovación: Emprender la tarea de ser autor y no sólo actor de sus propios proyectos. Revista de Investigación en Educación, 10(1), 7-29. http://reined.webs.uvigo.es/index.php/reined/article/view/131/121

Huber, G. L. (2008). Aprendizaje activo y metodologías educativas. Revista de Educación, número extraordinario 1, 59-81. http://reforma.fen.uchile.cl/Papers/Active\%20learning\%20 and $\% 20$ methods\%20of\%20teaching\%20-\%20Huber.pdf

Ilter, İ. (2014). A study on the efficacy of project-based learning approach on social studies education. Educational Research and Reviews, 9(15), 487-497. https://doi.org/10.5897/ ERR2014.1777

Jiménez Frías, R. A., Gómez Rodríguez de Castro, F., Aguado Odina, M. T. y Ballesteros Velázquez, B. (2001). Cuéntame: El cuento y la narración en educación infantil y primaria. UNED.

Johnson, A. E. (2008). Digital ink: In-class annotation of PowerPoint lectures. Journal of Chemical Education, 85(5), 655-657. https://doi.org/10.1021/ed085p655

Martínez-Bello, V., y Bernabé Villodre, M. del M. (2019). Experiencia universitaria innovadora sobre didáctica de la motricidad infantil: De las aulas universitarias a las aulas de Educación Infantil. Revista Electrónica Educare, 23(3), 1-26. http://dx.doi.org/10.15359/ree.23-3.15

Monzón, J. (2011). Las prácticas inclusivas en los servicios de apoyo externos a la escolaridad de alumnado con necesidades educativas especiales en el País Vasco. Revista Latinoamericana de Educación Inclusiva, 5(2), 189-203. http://www.rinace.net/rlei/numeros/vol5-num2.html

Navaridas Nalda, F. (2004). Estrategias didácticas en el aula universitaria. Universidad de la Rioja, Servicio de publicaciones.

Núñez Mayán, M. T. (2008). Da segregación á inclusión educativa. Galicia: Edicións Laiovento.

Orcasitas, J. R. (2005). 20 años de integración escolar en el País Vasco: Haciendo historia..., construyendo un sistema educativo de calidad para todos. En La respuesta a las necesidades educativas especiales en una escuela vasca inclusiva. Actas del congreso Guztientzako Eskola (pp. 37-93). Gobierno Vasco, Servicio de Publicaciones. http://www.hezkuntza. ejgv.euskadi.eus/r432459/es/contenidos/informacion/dia6/es 2027/adjuntos/escuela inclusiva/Respuesta necesidades c.pdf 
http://doi.org/10.15359/ree.24-3.11

http://www.una.ac.cr/educare

educare@una.ac.cr

Pazos Couto, J., Venegas Rojas, M., Abelairas Gomez, C. y Cons Ferreiro, M. (2015). La construcción del conocimiento desde la vivencia/experiencia del alumnado de la materia educación. Desenvolvimiento motor en la formación del docente de educación infantil. Estudios Pedagógicos, 41 (especial), 181-192. https://doi.org/10.4067/\$0718-07052015000300012

Perroca, M. G. (2011). Desarrollo y validación de contenido de la nueva versión de un instrumento para clasificación de pacientes. Revista Latino-Americana de Enfermagem 19(1), 58-66. http://dx.doi.org/10.1590/S0104-11692011000100009

QSR International. (2011). NVivo9 software. Autor. https://www.qsrinternational.com/nvivo/ support-overview/downloads

Quintana Albalat, J. (2000). Competencias en tecnologías de la información del profesorado de educación infantil y primaria. Revista Interuniversitaria de Tecnología Educativa, 166-174. http://www.ub.edu/ntae/jquintana/articles/competeTIC 2000.pdf

Robinson, V. (1993). Problem-based methodology: Research for the improvement of practice. Pergamon Press.

Rojas, F. J., Cepero, M., Zurita, F. y Chinchilla, J. L. (2015). Simulación del desempeño profesional, recurso didáctico en docencia de ciencias del deporte. Revista Internacional de Medicina y Ciencias de la Actividad Física y del Deporte, 57, 17-28. https://doi.org/10.15366/ rimcafd2015.57.002

Tárraga Mínguez, R., Martínez Monfort, P., Gómez del Amo, R., Pastor Cerezuela, G. y Fernández Andrés, I. (2012). Análisis de las preferencias instruccionales de estudiantes universitarios. El papel del material de apoyo a las clases. IE Comunicaciones, 16, 69-77. https://dialnet. unirioja.es/servlet/articulo?codigo $=4095330$

Tejada Fernández, J. (2010). La evaluación de las competencias en contextos no formales. Dispositivos e instrumentos de evaluación. Revista de Educación, 354, 731-745. 10.4438/1988-592X-RE-2011-354-018

Urra Medina, E. Sandoval Barrientos, S. e Irribarren Navarro, F. (2017). El desafío y futuro de la simulación como estrategia de enseñanza en enfermería. Investigación en Educación Médica 6(22), 119-125. https://doi.org/10.1016/j.riem.2017.01.147

van Dijk, T. A. (1996). La ciencia del texto. Barcelona. Paidós. 